\title{
Buenas Prácticas en Medidas y Programas para Jóvenes Desenganchados de lo Escolar
}

\author{
Best Practices for Measures and Programs for School \\ Disengaged Young People
}

\author{
M ${ }^{\mathrm{a}}$ Teresa González González ${ }^{1 *}$ \\ José Luis San Fabián ${ }^{2}$ \\ ${ }^{1}$ Universidad de Murcia, España \\ ${ }^{2}$ Universidad de Oviedo, España
}

\begin{abstract}
El desenganche de ciertos estudiantes con el aprendizaje escolar está estrechamente relacionado con el absentismo, el abandono temprano y las consecuencias educativas y socio-laborales que lo acompañan. Para contrarrestarlo, se promueven y desarrollan diversas medidas y programas. El artículo ofrece una visión panorámica de estas "buenas prácticas" documentadas por la investigación. Para ello se ha realizado una revisión de bibliografía de investigación en la última década sobre programas de reenganche y orientada a paliar el abandono. Los resultados indican que la atención y el cultivo de climas relacionales de respeto y cuidado, un currículo relevante y significativo, dinámicas de enseñanza centradas en los estudiantes, cuidada relación con otras entidades de la comunidad, coordinación y colaboración entre profesionales, flexibilidad de programas, tiempos, espacios y estructuras son, entre otras, prácticas prometedoras para trabajar con alumnado desenganchado y en riesgo de abandono. El conocimiento sobre rasgos de calidad que caracterizan a las prácticas de reenganche documentadas como exitosas en términos de posibilitar la persistencia y evitar abandonos, constituye un referente relevante de cara a analizar programas existentes y cómo discurren en la práctica, y plantear mejoras que alteren rutinas y modos de hacer asentados, que están en la raíz de procesos de desenganche.
\end{abstract}

Descriptores: Deserción escolar; Práctica pedagógica; Relación estudiante-escuela; Programas de educación; Educación.

\begin{abstract}
The student disengagement is closely related to early school dropout and its educational, social and labour consequences. The measures and programs aiming to counteract this problem are diverse. This paper offers an overview of those good practices that are documented in the research literature. To do so, it has been made a literature revision of research from the last decade about programs and measures to relieve the school dropout. The results shown cultivating a respectful and caring atmosphere, a relevant and meaningful curriculum, student-centered teaching practices, a close relationship with other organizations in the community, the professional coordination a collaboration as well as program, timetables, spaces and organizational flexibility are, among others, some promising practices for working with disengaged and at-risk students. Knowledge about quality features of reengagement programs that are documented by the research as successful at favoring the persistence and preventing school dropout are a reference to analyze the current programs and their implementation, and to suggest improvements aimed to modify established routines that lie at the root of school disengagement.
\end{abstract}

Keywords: Dropping out; Teaching practice; School student relationship; Educational programmes; Education.

*Contacto:mtgg@um.es

ISSN: 1696-4713

www.rinace.net/reice/

revistas.uam.es/reice
Recibido: $\quad 15$ de diciembre 2017

$1^{\text {a }}$ Evaluación: 22 de enero 2018

$2^{\text {a }}$ Evaluación: 15 de febrero 2018

Aceptado: $\quad 14$ de febrero 2018 


\section{Introducción}

El presente artículo pretende ofrecer una síntesis de algunas de las prácticas pedagógicas que, según diversas investigaciones consultadas, son prometedoras a la hora de trabajar con estudiantes desenganchados de la educación y tratar de evitar su abandono escolar, posibilitando de ese modo que persistan en su formación. El contenido del texto está, pues, indirectamente ligado a un fenómeno que genera en nuestros días una amplia preocupación en el ámbito educativo -ya sea en el plano de las políticas educativas estatales y autonómicas o locales, ya en los propios centros escolares-, como es el del abandono escolar temprano. Se aborda desde la consideración de que este es el resultado o punto final de un proceso progresivo de desafección y pérdida de interés de ciertos estudiantes por lo escolar y lo académico, que evoluciona a medida que transitan por la institución escolar hasta el abandono definitivo.

Un estudiante que, por las causas y factores que fuere, va perdiendo su interés por lo escolar y por su formación, es un alumno en riesgo de abandono. Los centros escolares, de acuerdo con las políticas del sistema educativo del que forman parte, suelen desarrollar y poner en práctica diversos tipos de medidas y programas, ya sean preventivos o paliativos, de lucha contra el abandono, en orden a aminorar ese riesgo. Constituyen caminos o vías destinadas a asegurar la persistencia de los estudiantes en el contexto escolar, o para reengancharlos o reincorporarlos en la educación o en otras actividades formativas que le permitan transitar al mundo socio-laboral.

El foco de este artículo se sitúa, precisamente, en tales programas y medidas. Son múltiples y de diversa naturaleza, existiendo investigación sobre ellas, tanto en contextos de la Comunidad Europea y los distintos Estados Miembros como en contextos estadounidenses, canadienses o australianos, entre otros. Aunque es notable su amplitud y variedad, existe ya un corpus suficiente para identificar "buenas prácticas". Esta expresión se utilizará aquí para aludir a distintas acciones, relaciones, dinámicas pedagógicas y climas de aprendizaje que se propician y se ponen en juego en los programas o medidas más exitosas. Es este artículo, con tal calificativo se alude a aquellos que han mostrado mejores resultados en términos de reenganche o persistencia del estudiante en su formación y que, por tanto, han contribuido a evitar el abandono prematuro definitivo. Es cierto que este es, entre otros, uno de los criterios que cabe barajar a la hora de hablar de éxito de programas y medidas de esta naturaleza, pero sin duda puede considerarse como un requisito para el logro de otras metas deseables asociadas al mismo, como son la adaptación social, la integración laboral o la formación ciudadana.

El contenido de las páginas que siguen se ha elaborado a partir de una revisión de la literatura -realizada en el marco de un proyecto de investigación más amplio ${ }^{1}-$ sobre desenganche escolar de los estudiantes y los programas o prácticas pedagógicas para paliarlo y evitar el abandono prematuro.

Teniendo en cuenta que el alumnado desenganchado no es homogéneo, y de que las acciones prácticas necesariamente están ligadas a contextos y circunstancias escolares y sociales particulares, en las páginas que siguen se ofrecerá una panorámica general de aquellos aspectos que destaca la literatura en lo que respecta a prácticas educativas y ${ }^{1}$ Proyecto I+D (2016). "Procesos de reenganche educativo y sociolaboral de adolescentes en situación de
vulnerabilidad. Estudio de casos e implicaciones socioeducativas". Referencia: EDU2016-76306-C2-1-R. 
entornos de aprendizaje con estudiantes en riesgo, potencialmente exitosos y que cabría considerar nucleares a la hora de proponer medidas de reenganche educativo o formativo.

El texto se ha estructurado en tres apartados. En el primero se bosqueja el marco teórico desde el que se ha planteado la revisión bibliográfica a la que se alude en este artículo. El segundo refiere, muy escuetamente, el procedimiento seguido para realizar tal revisión. En el tercero, se comentan las principales prácticas o patrones de funcionamiento que se han ido constatando como valiosos en procesos de reenganche o de aminoración de posibilidades de abandono escolar.

\section{Marco teórico}

En este primer punto se presenta brevemente el marco teórico desde el que se ha realizado la revisión bibliográfica recogida en las páginas que siguen. Se ha estructurado en dos apartados; en el primero se hace una primera alusión a la relevancia e incidencia del desenganche educativo, la preocupación manifestada por numerosos países y organismos internacionales por tal fenómeno y la abundante investigación que se ha ido desarrollando sobre sus distintas facetas y manifestaciones en los últimos años, tanto en el contexto español como internacional. En el segundo, se adelanta y explica que el foco de la revisión efectuada se sitúa básica y preferentemente en investigaciones referidas a la puesta en práctica exitosa de programas y medidas para abordar y paliar dichos problemas.

\subsection{Desafección escolar y abandono: Un tema que preocupa}

El fenómeno de la desafección o desenganche de los aprendizajes y la vida escolar por parte de ciertos alumnos y, ligado a él, el abandono prematuro; el nada desdeñable número de estudiantes sin titulación que les impide proseguir su educación o insertarse en el mundo laboral; el aumento de jóvenes que habiendo abandonado sus estudios no están realizando ninguna actividad formativa pero tampoco de carácter laboral, o de jóvenes que aunque no han abandonado cuentan con una trayectoria escolar muy irregular y un acusado fracaso escolar, constituye un foco de preocupación en nuestra sociedad. No es de extrañar que sea así en momentos como los actuales, en los que la presión por los buenos resultados de los estudiantes está al orden del día, y en los que se sabe que quienes abandonan temprano quedan en desventaja en el mercado de trabajo, con menor probabilidad de encontrar empleo a tiempo completo, y afrontan mayores dificultades sociales, económicas, de salud y bienestar en general (Rumberger, 2011; Sodha y Guglielmi, 2009). Se ha insistido y advertido, en ese sentido, de las nefastas consecuencias del desenganche y posterior abandono escolar prematuro en el desarrollo económico y competitividad de los países, como queda patente, por ejemplo, en informes y normativas de la Comunidad Europea y en los de sus estados miembros, incluidos el español (Comisión Europea, 2013, 2014; MECD, 2014, entre otros). Paralelamente, y denunciando lecturas puramente economicistas (Escudero, 2016; Rambla y Fontdevilla, 2015), también se ha venido insistiendo en que el riesgo de exclusión educativa y social que conllevan los fenómenos de desenganche y abandono escolar no está distribuido igualitariamente sino mediatizado por factores sociales, económicos y estructurales, más acusados entre poblaciones marginadas y vulnerables.

En cualquier caso, es comprensible que el poner en marcha medidas tendentes a evitar el abandono (último peldaño del proceso de desenganche) o a posibilitar la vuelta y reenganche, sea una cuestión de creciente interés subrayada en las agendas de inclusión 
social internacional y priorizada en los niveles estatales y autonómicos a través de distintas políticas de reforma educativa.

Abordar este problema constituye un reto importante en los sistemas educativos europeos y, en general, de la oCDE. Un claro ejemplo es el español, con tasas de fracaso y abandono escolar notables, en el que es evidente el afán de regular líneas de actuación y medidas de lucha contra el abandono que frenen y eviten el aumento de jóvenes que se descuelgan de la vida escolar para finalmente no proseguir su formación. Una muestra de esta preocupación es el interés por investigar y comprender en todas sus dimensiones dicha problemática, y elaborar conocimiento y propuestas de actuación al respecto, como puede colegirse de distintos análisis sobre abandono escolar (Fernández Enguita, Mena y Riviére, 2010; Mena, Fernández Enguita y Riviére, 2010; Tarabini, 2015, entre otros) o en torno a diferentes medidas para evitar fracaso escolar en la Educación Secundaria Obligatoria y, por tanto, el abandono "forzoso" que se deriva del mismo (p. ej. Escudero, 2013, 2016; González, 2015a, 2016).

Referencias como las anteriores se localizan en el contexto del estado español, pero el asunto está al orden del día en otros muchos países, entre ellos, y por citar sólo algunos más próximos, los del entorno europeo, como refleja la amplia investigación disponible sobre el tema (p. ej., Ashwin y McVitty, 2016; Blaya y Bergamaschi, 2015; CEDEFOP, 2010; Davies, Lamb y Doecke, 2011; Day, 2013; Evans et al., 2009; Kettlewell et al., 2012; Magalhaes et al., 2015; Nelson, 2011; Nelson y O’ Donell, 2012; Sodha y Guglielmi, 2009; Tomaszewska-Pekala, Marchlik y Wrona 2015; Traag y van der Velden, 2008; Trowler y Trowler, 2010; Wogboroma, 2014).

\subsection{La puesta en práctica de medidas y programas de reenganche}

Los programas y medidas que se han ido arbitrando desde las políticas educativas de diferentes países para afrontar los procesos de desafección y revertirlos son diversos. Igualmente, la investigación existente sobre las mismas -como se irá refiriendo en las páginas que siguen-es amplia en sus focos de atención: desde el análisis de las coordenadas sociopolíticas, económicas y educativas más generales en las que se promueven, pasando por las condiciones organizativas para llevarlas a cabo, los profesionales implicados y su formación, las conexiones con entornos comunitarios, empresariales, etc. hasta las prácticas pedagógicas concretas que se despliegan en las aulas y los modos en que las viven los estudiantes, los resultados académicos, personales y sociales, así como niveles de implicación y reenganche alcanzados por los destinatarios. Son planos de análisis complementarios y necesarios para conocer en profundidad qué está ocurriendo en los procesos de reenganche de esos jóvenes, con qué nivel de éxito -reinserción educativa y/o socio laboral- y qué prácticas pedagógicas están contribuyendo a ello.

Desde una perspectiva de intervención educativa, nos interesa aquí la investigación acerca de qué ocurre en la práctica con las medidas y programas destinados al reenganche escolar o formativo. El foco en centros y aulas no ha sido preeminente en este terreno del abandono y el desenganche, predominando enfoques de investigación más centrados en los estudiantes individuales y sus características o en sus entornos socioculturales, familiares y económicos, que en los marcos escolares en los que discurre su experiencia educativa. Aunque más escasa, tal investigación ha evidenciado que diversas dimensiones y dinámicas de funcionamiento curricular y organizativo de los centros educativos estarían en la raíz de porqué ciertos estudiantes, ante una experiencia escolar que no les resulta significativa ni satisface sus necesidades e intereses, fracasan e, incluso, abandonan 
(Aristimuño y Parodi, 2017; Bond et al., 2007; Escudero y Martínez, 2012; González, 2015b; Knesting, 2008; McMahon et al., 2012; Morentin y Ballesteros, 2018; Salvà, Trobat, y Forgas, 2014; Seguedin, 2012; Strobel et al., 2008); igualmente, ha puesto de manifiesto cómo distintas razones aducidas por los estudiantes para justificar su desenganche están relacionadas con la experiencia educativa que le ofrece el propio centro escolar: modos en que están estructuradas las clases, el aburrimiento en ellas y la escasa actividad práctica, las escuetas o tirantes relaciones con los profesores, el currículo al que se enfrentan, el clima rígido y punitivo, la falta de apoyo, y un largo etcétera (p. ej., Mills y Mcgregor, 2010).

En sentido contrario, la investigación que se revisa aquí sobre la puesta en práctica y desarrollo de estrategias frente a la desafección escolar y el abandono que resultan exitosas, muestra que, cuando confluyen las condiciones y prácticas oportunas, lo que ocurre en el centro escolar y las aulas deja de ser un factor de riesgo para ser un factor de protección y prevención frente a desenganches y abandonos.

Mirar al funcionamiento organizativo y educativo de los centros y aulas para explorar en qué medida están contribuyendo a que algunos se descuelguen y abandonen o, mejor aún, indagar en aquellas iniciativas y medidas que contribuyen a una mayor implicación de los alumnos en sus aprendizajes constituye un conocimiento valioso que nos aporta la investigación sobre factores, condiciones y prácticas que pueden estar incidiendo en el abandono y, en consecuencia, posibilitar el reenganche educativo.

\section{Método}

La revisión bibliográfica sobre los procesos de desenganche escolar y puesta en práctica exitosa de programas y medidas para revertirlo, se ha realizado a través de la consulta de informes, artículos u otros documentos (resúmenes estadísticos, tesis doctorales, informes oficiales de evaluación, etc.). Buena parte de ellos se localizaron a través de Internet, fundamentalmente en Google o, para algunas búsquedas más específicas en Google Académico. En todo momento se tomó como criterio de selección básico que se tratase de documentación de carácter académico y referida a investigaciones sobre programas y medidas desarrolladas en organizaciones educativas. Se descartaron, pues, textos meramente divulgativos, periodísticos, comentarios en blogs, copias de presentaciones en power point y similares. Los términos básicos de búsqueda fueron: enganche, desenganche, desafección, desconexión, desvinculación y segunda oportunidad utilizados en español y en su correspondiente traducción en inglés, combinados con los términos escolar, aprendizajes, educativo, estudiantes. Se ha revisado preferentemente documentación a partir del año 2007, con algunas excepciones, por su relevancia, de artículos o documentos anteriores.

Aunque no es el objetivo de la presente investigación, es relevante señalar dos primeras conclusiones que surgen de la búsqueda y filtración de documentación:

- No es posible ni pertinente hablar en general de "alumnos desenganchados": estamos ante un contingente de estudiantes muy heterogéneo en lo que respecta tanto a sus características sociales, personales y culturales como a su grado o nivel de desenganche, que puede ser de distinto calado, siéndolo también, por tanto, sus intereses y sus necesidades educativas (Callahan et al., 2009; Evans et 
al., 2009; Nelson y O’Donnell, 2012; Ross 2009; Shoda y Guglielmi,2009; Spielhofer et al., 2009; Stamou et al., 2014).

- En consecuencia, no cabe hacer generalizaciones o pensar en posibles "fórmulas" salvadoras para un problema tan complejo, multidimensional y multifactorial, que requerirá vías de solución necesariamente contextualizadas, integradas y buenas prácticas que tengan en cuenta distinciones y matices diversos.

\section{3. Ámbitos de buenas prácticas en medidas y programas frente al desenganche y el abandono escolar}

La investigación revisada pone de manifiesto cómo las prácticas que caracterizan a los programas y medidas que generan buenos resultados tocan múltiples aspectos, desde los curriculares, a otros más estructurales, relacionales, de clima y ambiente de aprendizaje, de apertura y relación con la comunidad, etc. poniendo de manifiesto la importancia de mantener una mirada multidimensional. En los siguientes sub-apartados se hará una breve referencia a ellos. No se trata, pues, de prácticas o modos de actuar aislados unos de otros, sino, al contrario, interrelacionados y complementarios.

\subsection{Atender a aspectos relacionales}

Las relaciones y conexiones de los estudiantes con docentes, apoyos y compañeros, su sentido de pertenencia y los vínculos socio afectivos en su entorno escolar ocuparon siempre un lugar relevante en la literatura sobre engagement (Conner, 2016). Se ha venido considerando como una de las dimensiones que, junto con la conductual y la cognitiva, configuran la implicación del alumnado en sus aprendizajes (González, 2010).

Lo relacional, en el sentido más amplio del término, cobra especial importancia en el análisis de los procesos de desenganche escolar. Atweh y otros (2007), por ejemplo, planteaban hace una década la pertinencia de concebirlo como un proceso de ruptura o fracaso de las relaciones entre estudiante y escuela. Por su parte, Zyngier (2014) mantiene que la implicación (engagement) de los jóvenes con su formación requiere que haya relación entre esta y la vida cotidiana del alumno, subrayando la importancia de que se sientan conectados e implicados en sus propias comunidades y de una pedagogía que cultive y alimente tales conexiones. También Smyth y otros (2008) reconocen la importancia de los componentes relacionales desde lecturas amplias y no únicamente focalizadas en el alumno particular. A partir de su investigación concluyen que "el resultado más profundo... fue que los jóvenes renuncian a la escuela cuando no pueden formar relaciones sostenibles con pares, adultos y desde luego la propia institución" (p. 2). Esa faceta relacional, apuntan los autores, alude tanto a relaciones interpersonales como a relaciones ideacionales. Las primeras, relativas a las desarrolladas con los estudiantes, que habrían de estar basadas en el respeto, la confianza, el cuidado y consideración hacia el otro. Las segundas, referidas a cómo se inicia a los jóvenes en el proceso de relacionarse con ideas y asuntos sociales más amplios e importantes "cómo las analizan, cómo interrogan la información, hacen transferencia de conocimiento, y desarrollan posiciones éticas respecto a cuestiones socialmente importantes sobre las que adoptar con prudencia y coraje una postura" (pp. 158-159). Ambos aspectos, concluyen los citados autores, son relevantes y cruciales de cara a enganchar a los estudiantes en la escuela y sostener su deseo de querer continuar el aprendizaje formal. 
Aunque aportaciones como las indicadas reflejan la coexistencia de diferentes concepciones sobre los aspectos y facetas relacionales ligados al enganche o desenganche de los estudiantes con sus aprendizajes, buena parte de la bibliografía revisada (Bond et al., 2007; Callanan et al., 2009; Ingólfur y Valgerður, 2016; Ketewell et al., 2012; Knesting, 2008; Li, 2017; Maroulis, 2008; McLaughlin y Gray, 2015; Murray-Harvey y Slee, 2007; Nouwen et al., 2016; Ross, 2009; Rumberger et al., 2017; Suárez, Pimentel y Martín, 2009), particularmente cuando alude a resultados positivos de programas y medidas de reenganche, destaca y pone sobre la mesa el papel que en procesos de reenganche desempeña el cultivar atmósferas o climas relacionales de atención y "cuidado" personalizado, así como las relaciones positivas mutuas, de respeto y colaboración entre los implicados.

Si bien este ámbito abarca relaciones con compañeros, directivos, otros agentes de la comunidad, etc. nos referiremos aquí especialmente a las establecidas entre alumnos y docentes u otros profesionales en los programas o medidas de reenganche, a las que aluden comúnmente los jóvenes cuando explican qué motiva su falta de interés en lo escolar, o su decisión de abandonar. No se circunscriben únicamente al aula o los espacios formativos en que transcurra un determinado programa o medida, sino también al centro en su conjunto. Aportaciones de los investigadores antes referidos han destacado la relevancia de cultivar en el centro un clima social y académico personalizado y acogedor frente a uno inhóspito, despersonalizado, burocrático y de anonimato. Si el primero está relacionado con el desarrollo por los estudiantes de un sentido de pertenencia al centro escolar y mayor intención de permanecer e implicarse, el segundo estaría contribuyendo a su desenganche, así como a un menor rendimiento.

Interacciones con los docentes que contribuyen a que los estudiantes puedan vivir el centro, el aula u otros espacios de formación como entornos de los que forman parte, a los que pertenecen, en los que pueden participar e implicarse... estarían ligadas a prácticas de aula en las que se presta atención cuidadosa a conocer a los estudiantes como personas totales, a una atmósfera de relaciones de confianza, respetuosas y de apoyo; el escucharlos y respetar su conocimiento; estar al tanto de cómo evolucionan académicamente, con qué dificultades se están encontrando y, por tanto, qué apoyos y respuestas curriculares pueden necesitar; el conectar con sus vidas, sus propios intereses y reconocer sus voces; el potenciar la interacción significativa entre estudiantes en el contexto del aula y las tareas escolares más que patrones de trabajo individual y aislado. Elementos todo ellos relevantes. Como han señalado Hancock y Zubrick (2015):

$$
\begin{aligned}
& \text { La formación de relaciones es un aspecto central para el enganche de los estudiantes. } \\
& \text { Sin esto, la excelencia en la pedagogía, la flexibilidad curricular, y las políticas, } \\
& \text { aunque necesarias, no serán suficientes para reenganchar a alumnos desenganchados } \\
& \text { o que se están desenganchando. (p. 9) }
\end{aligned}
$$

\subsection{El currículo y los modos de desarrollar la enseñanza}

La literatura revisada insiste en que la falta de implicación y consiguiente desenganche no es un fenómeno ajeno al currículo que se oferta en el centro: los estudiantes no pierden el interés y se desenganchan de los aprendizajes en general sino de aquellos que promueve el currículo escolar. Esta situación se produce incluso en programas específicamente pensados para alumnos desenganchados (González y Porto, 2013).

El tema del currículo y su papel en los procesos de desafección y abandono escolar es complejo: qué currículo, qué estructura curricular, cuánto peso ha de tener lo académico o 
lo práctico, el cultivo de qué competencias para afrontar las complejidades del mundo actual e, igualmente, qué enseñar, con qué metodologías, cómo asegurar experiencias escolares de calidad y aprendizajes valiosos para el alumnado desafectado o que ya ha abandonado y retoma de nuevo su formación y educación, son cuestiones habituales en la bibliografía y documentación revisada.

No es un asunto libre de polémicas, particularmente en lo que respecta al peso que debe tener la formación académica y la de carácter más práctico o profesional (Nelson y O’Donell, 2012), o a la atención curricular que también -ante niveles de desenganche muy acusados y propiciados por barreras y dificultades en la vida cotidiana de los estudiantescabe prestar a las habilidades "blandas" como el cultivo de la autoestima, lo emocional y relacional, las habilidades para la vida cotidiana, etc. (Biebly et al., 2002; Evans et al., 2009; Ketewell et al., 2012). Alargaría en exceso el artículo entrar aquí en todos estos aspectos; sólo se hará una breve alusión a algunos rasgos curriculares más destacados en la investigación e informes revisados sobre el tema que nos ocupa (ALA, 2010; Kettellew et al., 2012; Lloyd-Jones et al., 2010; Lumby, 2013; Mills y McGregor, 2010; Murray y Mitchell, 2016; Portelli, Shields y Vibert, 2007; Smyth, 2012; Smyth et al., 2008; Tadich et al., 2007; Zyngier, 2007, 2012).

Un aspecto comúnmente referido alude a las mayores posibilidades de reenganche o de paliar desenganches cuando el currículo es considerado y planteado como espacio para explorar la relación entre los diferentes materiales y conocimientos que se estén trabajando con los alumnos/as y sus propias experiencias e intereses. En otros términos, no sería propicio en procesos de reenganche un currículo planteado como conocimiento acreditado a trasmitir a los alumnos para que lo reproduzcan y poco más. Más bien, el éxito de medidas de reenganche escolar estaría ligado a un currículo:

- Que posibilite aprendizajes que estén conectados a la vida de los jóvenes, organizado alrededor de sus intereses vitales, experiencias y aspiraciones, con relevancia y significado para ellos. Es pertinente, pues, que las propuestas curriculares no pasen por alto quiénes son los estudiantes, qué saben, qué les interesa... cómo es la comunidad en la que viven, qué hacen, cómo es su vida, su cultura. También lo es tomar en cuenta su voz: el aprendizaje no se construye para ellos, sino con ellos.

- Que se lleve a cabo de modo integrado, más que segmentado en asignaturas o módulos poco relacionados entre sí. Un currículo que facilite articular el trabajo con los estudiantes en torno a problemas reales cuya resolución requiere echar mano de aportaciones provenientes, integradamente, de diferentes asignaturas o ámbitos de conocimiento teórico y práctico.

- Que implique a los estudiantes en tareas rigurosas, auténticas y socialmente valiosas. Un currículo superficial y poco riguroso es, como apuntan algunos (Riele 2014; Zyngier y Gale, 2003), no equitativo.

- Que, cuidando esa conexión con las experiencias y conocimientos del estudiante, no se queda en la propia experiencia, sino que contribuye a interrogarla y desafiarla, a desarrollar en todos los estudiantes disposiciones, modos de pensar y aprendizajes necesarios para interpretar críticamente el mundo en el que vive cotidianamente (Zyngier, 2007). 
Correlativamente, la investigación consultada también aporta información sobre los modos y patrones de enseñanza en las aulas que estarían resultando en un mayor o menor éxito en revertir el desenganche o en lograr un reenganche educativo o formativo.

A nadie se le escapa que las prácticas concretas que pueden contribuir a que los alumnos se impliquen y enganchen no tienen un carácter universal, pues están ligadas a los contextos concretos en los que ocurren sin que sean fácilmente transferibles a otros de manera directa. Tomando en cuenta esa advertencia, se comenta a continuación, a título ilustrativo, algunos rasgos característicos de procesos de enseñanza a los que se alude frecuentemente en la literatura como favorecedores del reenganche o reincorporación y persistencia de los estudiantes. Entre otros se documentan como exitosos experiencias y programas en los que:

- La enseñanza es más personalizada, cuidando que las respuestas pedagógicas estén ajustadas a necesidades e intereses de los estudiantes.

- Se echa mano de experiencias de aprendizaje valiosas y significativas, actividades de resolución de problemas del mundo real... Cultivo de habilidades superiores de pensamiento.

- Se pone en juego las diversas posibilidades que ofrecen las metodologías de aprendizaje colaborativo, basadas en la indagación, de carácter práctico, en la que alumnos investigan temas, cuestiones, asuntos de sus vidas y experiencias.

- Se atiende tanto a lo académico como a lo emocional y social del aprendizaje humano y escolar, y se refuerzan vínculos y relaciones -un sentido de comunidad- entre los estudiantes y entre estos y los docentes.

Elementos como los indicados ponen de manifiesto el papel que pueden desempeñar nuevos modos de desplegar el trabajo con los estudiantes en las aulas, marcadamente diferentes de los estilos de enseñanza autoritarios y chapados a la antigua, con poco diálogo y flexibilidad, cuyo uso, como señalan Fullan y Longworthy (2014), desmoraliza a los jóvenes.

Por su relación, cabe mencionar otros dos aspectos estrechamente relacionados con las dinámicas de trabajo en las aulas con los alumnos: uno, la importancia de los procesos de evaluación de los aprendizajes, no centrados únicamente en resultados académicos, en los que se ofrece feedback positivo, se valoran y reconocen logros como vía de apoyo al aprendizaje. Otro, sin duda muy relevante, como es el papel crucial de los pertinentes mecanismos de tutoría, de apoyo académico y personal, de orientación a los más desenganchados, en sus procesos de reenganche educativo y/o formativo (CEDEFOP, 2010; Day et al., 2013).

En síntesis, y sin ánimo de generalizar, en prácticas curriculares y de aula de programas de reenganche exitosos -en el sentido con el que se está utilizando aquí, el término exitoso- se cuida que los estudiantes puedan ver y vivenciar sus vidas y experiencias cotidianas como relevantes para su aprendizaje y éxito escolar, puedan sentir que se respetan los conocimientos y habilidades que traen consigo, y puedan implicarse en actividades de resolución de problemas del mundo real. En estas coordenadas, los componentes relacionales a los que se hacía referencia en el apartado anterior son clave. Así, se identifican como buenas prácticas aquellas en las que los docentes u otros profesionales se esfuerzan por: a) conocer y reconocer lo que los jóvenes hacen y son fuera del ámbito escolar en orden a hacer conexiones pedagógicas efectivas en el aula; b) 
ayudarles a explorar sus propios intereses personales y de su entorno y c) aportarles oportunidades para que puedan negociar aspectos de su aprendizaje y tomar en consideración sus experiencias fuera del centro escolar. La cercanía o lejanía entre estas y las promovidas en las instituciones educativas constituye, como ya se han indicado, un aspecto a considerar seriamente cuando hablamos del desenganche y no implicación de alumnos en la escuela.

\subsection{Conexiones y relaciones con organizaciones de la comunidad}

Se comentan en este apartado algunas prácticas relevantes destinadas a establecer y cultivar relaciones con organizaciones culturales, sociales, educativas, empresariales, etc. de la comunidad, en cuanto que fuente de oportunidades reales de aprendizaje-académicos o prácticos- más allá de las paredes del centro educativo.

Una de ellas es la de tomar en cuenta la comunidad, los entornos en los que viven los estudiantes, así como las barreras o dificultades que acompañan a aquellos que provienen de entornos complejos y de amplia vulnerabilidad. Wilson, Stemp y McGinty (2011) por ejemplo, insisten en aportaciones de investigadores que apuntan que el trabajo con alumnos cuyas experiencias de vida son muy complejas, alejados de la cultura tradicional de la escuela y en desventaja por su falta de capital social, cobra una gran relevancia el cultivo de relaciones de aprendizaje que "sean inclusivas de las familias y las comunidades de los estudiantes y, como tal, apoyen holísticamente y posibiliten a los jóvenes a construir capital social" (p. 33). Especialmente insistentes en el valor de las conexiones y relaciones con la comunidad son investigaciones que, desde planteamientos críticos y sociocríticos, han documentado programas y medidas de reenganche orientados por la idea de un "engagement comunitario", en el que las nociones ya comentadas, de estar conectado a, relevancia curricular, currículo cercano a la vida de los jóvenes, etc. ocupan un lugar nuclear.

Se trata de programas que promueven una mayor implicación y conexión con el mundo real, por tanto, con la comunidad a la que pertenecen los jóvenes destinatarios. Se desarrollan desde un enfoque de "pedagogía crítica del enganche que coloca a las escuelas como un recurso comunitario que puede trabajar en colaboración con los residentes locales para construir una comunidad mejor" (Smyth et al., 2008, p. 155). Se insiste, en esta línea, en la importancia de que los centros escolares generen los marcos adecuados para trabajar "colaborativa y democráticamente con sus comunidades e involucrarlas en las vidas educativas de sus estudiantes" (p. 152).

Por lo demás, en la literatura más al uso, que ya se ha ido citando en apartados previos, son diversas las actuaciones que se documentan como exitosas en medidas o programas de reenganche, entre ellas:

- La existencia de un planteamiento y política de centro sobre las relaciones con las familias y sobre cómo implicar a estas y a otras personas que puedan ser relevantes en la vida de los jóvenes desafectados de los aprendizajes escolares.

- El establecimiento de contactos, acuerdos y convenios con agencias, servicios sociales, empleadores o empresas locales. Constituyen una fuente de posibilidades para que programas y medidas de reenganche puedan ofertar oportunidades de experiencia laboral, prácticas y apoyos para actividades más allá del centro educativo. Igualmente, resulta relevante es el establecimiento de conexiones con otros centros escolares de cara a intercambiar información y 
conocimientos, experiencias de innovación y mejora relacionadas con estrategias de reenganche, etc.

Junto con elementos como los anteriores de apertura e interacción con la comunidad, también en la literatura revisada se insiste en prácticas que posibilitan y apoyan que los estudiantes aprendan, en contextos reales, con expertos externos al centro, profesionales de diferentes ámbitos, voluntarios, en otras organizaciones, haciendo uso de las oportunidades que ofrecen las tecnologías digitales (Paul Hamilton Foundation, 2012). En una línea similar, Smyth y otros (2008) defienden el uso de los "fondos de conocimiento" de la comunidad y documentan su utilización en medidas y programas exitosos de reenganche en los que la conexión y apertura a la comunidad ocupan un lugar relevante.

\subsection{Otras condiciones organizativas}

Aspectos como los comentados en apartados anteriores constituyen ejes importantes sobre los que se articulan programas, medidas educativas o formativas que han resultado exitosas en revertir el desenganche y desafección de estudiantes. Es bien sabido, no obstante, que difícilmente tales prácticas se podrán desarrollar si, al tiempo, no se cultivan desde los propios centros educativos ciertas condiciones y dinámicas de funcionamiento organizativo. Son estas las que, en última instancia, harán posible que principios y aspiraciones del centro o la institución educativa -en este caso, relativos a construir una organización que "enganche" a los alumnos en el aprendizaje- se materialicen en prácticas concretas y cotidianas. La literatura revisada no contempla como exitosas las actuaciones aisladas, desconectadas unas de otras sino, más bien, aquellas en las que se replantean todas las facetas y dimensiones que configuran la organización educativa como tal: las de carácter más pedagógico como también otras relacionadas con su vida o funcionamiento como organización. Algunas de las condiciones organizativas reiteradamente aludidas (Mills y McGregor, 2010; Menzies y Baars, 2015; Portelli, Shields y Vibert, 2007; Paul Hamilton Foundation, 2012; Riele, 2014; Smyth et al., 2008), sin entrar en detalles que alargarían el texto en exceso, son, entre otras, la presencia de:

1) Dinámicas de coordinación y colaboración entre docentes y otros profesionales implicados en programas y medidas de reenganche, evitando el trabajo fragmentado y en aislamiento. Es una condición imprescindible para desarrollar ese currículo relevante, próximo a las vidas y necesidades del alumnado, hacerlo con coherencia y continuidad, al que ya se aludió en apartados anteriores. Ello tiene que ver con cultivar y alimentar la disposición de los profesionales implicados en los programas o medidas a pensar y trabajar conjuntamente sobre aspectos relacionados con su práctica educativa, a cuestionar ideas, prácticas y rutinas tal vez poco ajustadas a las necesidades de esos alumnos y explorar vías de mejora. Establecer relaciones profesionales que permitan ir construyendo y manteniendo una concepción compartida sobre el programa o medida de re-enganche -en qué consiste, qué sentido tiene, porqué y para qué se imparte, sobre qué concepción de la formación, los aprendizajes, los alumnos..., sobre qué principios o "visión” se asientan las actuaciones que se van a llevar a cabo con los estudiantes...- colaborar en planificar el trabajo que se va a desarrollar en el programa y acordar estrategias pedagógicas básicas a utilizar, clarificar aspectos relativos a la evaluación de estudiantes y de la propia práctica, etc. Smyth y otros (2008) refiriéndose a su propio trabajo sobre experiencias con alumnado desenganchado en escuelas y comunidades en desventaja, apuntan al respecto: "Cuando las escuelas estaban comprometidas con crear espacios para la colaboración docente, la 
reflexión y planificación, la probabilidad de que hubiese posibilidades innovadoras para el enganche de los estudiantes fue mayor" (p. 134).

2) Un clima de centro/institución segura (sin amenazas, conflictos, riesgos de acoso...) y estable, en el que se sienten bien aceptados y bienvenidos ya en el centro en general o en los contextos de aula, prácticas, tutorías... en particular. Políticas de convivencia y de disciplina de naturaleza educativa y constructiva más que punitiva.

3) Condiciones que posibiliten la participación y voz de alumnos en las aulas y el conjunto del centro. Son claras a este respecto experiencias, programas y medidas a los que aluden, entre otros, Downem, Nairz-Wirth y Rusinaite (2016), Fielding (2012), Johns (2014), Menzies y Baars (201), Nowmen y otros (2016), Smyth (2006, 2012), Zyngier (2007), y la relevancia de cultivar principios democráticos en la institución educativa, dar a los estudiantes oportunidades $\mathrm{u}$ ofrecerles alternativas que les posibiliten ejercer poder, participar, disponer de cierta capacidad de actuación autónoma y expresar sus voces y puntos de vista. Como señalaban Echeita y Sandoval (2010, p. 13), "si es importante que los alumnos tengan oportunidades de participar en las decisiones que les afectan, especialmente lo es para los alumnos más vulnerables a la exclusión escolar".

4) Algunas condiciones estructurales y de carácter más formal como:

- Tamaños pequeños de aula y ratio alumnos-profesor bajas, que favorecen buena atmósfera/clima relacional pues permiten conocer a los alumnos mejor, estar con ellos el día completo..., o facilitan el acceso y cercanía de los alumnos a sus docentes.

- Mecanismos y procedimientos para la participación en decisiones del programa y su desarrollo en el aula, abordar temas de absentismo o relacionados con él, así como asuntos de comportamiento y disciplina.

- Procedimientos y criterios para establecer y sostener relaciones con las familias, otros centros e instituciones que desarrollan programas similares, o con los que se estén desarrollado planes de actuación conjuntos o en red, con centros de prácticas, asociaciones, servicios comunitarios, etc.

- Criterios pedagógicos para la distribución horaria (flexible) del trabajo de profesores y la actividad de los alumnos en el programa o medida en cuestión; para la organización y asignación de los espacios de trabajo y su rentabilización pedagógica; para la ubicación espacial de los programas, y aulas en que se desarrolla (Portelli, Shields y Vibert, 2007; Paul Hamlyn Foundation, 2012).

5) Previsión explícita y planificación de acciones de formación a realizar con los docentes y otros profesionales con los que se cuenta para desarrollar el programa(s) o medida(s).

6) Instalaciones, equipamiento y materiales adecuados y suficientes, facilitadores del trabajo y la labor docente y la tarea de los alumnos.

7) Mecanismos contemplados en el centro para recabar información sobre el programa o medida de re-enganche: su diseño, desarrollo e impacto que está teniendo en el alumnado, para analizarla y hacer uso de la misma en sucesivos procesos de toma de decisión y mejora de la práctica cotidiana con el alumnado más desenganchado o menos implicado en sus propios aprendizajes. 


\section{Consideraciones finales}

En los apartados previos se ha puesto de manifiesto la diversidad y complejidad del fenómeno del desenganche escolar y las prácticas constatadas como más exitosas al llevar a cabo medidas y programas arbitrados con la pretensión de paliarlo.

Estamos ante un panorama amplio y disperso de focos y líneas de investigación, así como de decisiones políticas en torno a un problema poliédrico sobre el que -como se apunta al inicio de este artículo- existe una gran diversidad de teorías, modelos y enfoques en torno a su comprensión y su resolución. Tal dispersión exige un ejercicio de síntesis y estructuración para fijar unas coordenadas que ayuden a familiarizarse con el conocimiento disponible, a orientarse dentro de este ámbito, a enmarcar y acotar debidamente las cuestiones y problemas y a emprender iniciativas sobre la base de lo ya sabido y realizado. En concreto, se han identificado las características más notables de los programas y medidas desplegadas frente al desenganche y el abandono escolar, Son características que implican diferentes niveles de intervención, desde el curricular al de la sociedad en su conjunto, si bien la revisión bibliográfica se ha centrado preferentemente en los niveles meso (centro escolar) y micro (aulas).

Siendo conscientes de las limitaciones inherentes a la pretensión de realizar una revisión bibliográfica sobre un asunto tan complejo y con tantas facetas, lo expuesto en apartados anteriores nos permite una primera y cautelosa aproximación a un conjunto de rasgos de calidad que caracterizan a las prácticas inclusivas de reenganche. Entre ellos los aspectos relacionales y de interacción educativa personalizada, respetuosa y emocionalmente rica; un enfoque curricular más apropiado, basado en el desarrollo de aprendizajes vitales, la experiencia e implicación del joven en su aprendizaje y la globalización, entre otros, lo que se traduce en patrones de enseñanza y entornos de aprendizaje alternativos a los habituales y en modos de valorar y reconocer logros como vía de apoyo al aprendizaje. También resulta fundamental el compromiso de la comunidad con las medidas de reenganche, sin olvidar determinadas condiciones organizativas, formales y no formales, de las instituciones facilitadoras.

Además de los anteriores aspectos, también debemos hacer referencia a programas exitosos fuera de los contextos escolares formales -escuelas de segunda oportunidad (Day et al., 2013; Gasch y Navas, 2017; Thureau y Usón, 2017); programas alternativos (Riele, 2014) - que revelan una atención cuidada a planes de aprendizaje personalizados; cultivo de habilidades de empleo, de habilidades para la vida y participación en experiencias de trabajo como parte integrante de la oferta formativa; reconocimiento del potencial de aprendizaje de actividades de distinta naturaleza, no sólo las académicas; y, en general, la importancia de desarrollar enfoques de intervención integral y holística. Investigadores en este ámbito exploran y valoran la eficacia de estas opciones formativas para satisfacer necesidades de jóvenes que rechazan lo ofertado por el centro escolar y que, por razones diversas, es improbable que vuelvan a él. Insisten en que los programas y medidas desarrolladas en contextos escolares regulares podrían aprender y beneficiarse de esas prácticas y modos de trabajo más flexibles y alternativos (Lago et al., 2015).

En todo caso, al igual que se ha evidenciado que el desenganche está ligado, entre otros aspectos, a la experiencia educativa vivida por el estudiante en su trayectoria escolar, la investigación sobre medidas de reenganche también constata que es la presencia de unas determinadas prácticas de aula, y ciertos entornos de aprendizaje, lo que contribuirá a que 
esas medidas sean una vía real de reenganche a la educación formal o a la formación para la transición al mundo del trabajo.

$\mathrm{El}$ conocimiento sobre el particular es relevante de cara a analizar programas existentes y cómo discurren en la práctica, plantear mejoras e incluso diseñar reformas. También en el marco de la gestión del conocimiento, una vía ampliamente utilizada para contribuir al aprendizaje y mejora de los programas existentes consiste en revisar las experiencias de éxito y buenas prácticas que están posibilitando el reenganche en la educación o la formación (Coffield y Edward, 2009).

El artículo ha tratado de aportar una visión general e introductoria al desenganche educativo con la finalidad de poner de manifiesto su importancia, su complejidad y, lamentablemente, su persistencia. Como visión panorámica, no se ha detenido en un análisis minucioso de la investigación sobre desarrollo de los múltiples programas y condiciones asociados a los procesos de reenganche. Pero puede ser una invitación a vislumbrar y emprender nuevas líneas de investigación que amplíen nuestro conocimiento sobre lo que realmente ocurre en la práctica con las diversas medidas y programas existente para desarrollar con el alumnado en situación de desenganche y riesgo de abandono, que permitan revisar las actuales políticas educativas dirigidas a reducir el fracaso escolar y que contribuyan a mejorar las actuaciones en centros, entidades formativas, aulas, en definitiva, con el alumnado. Algunas de estas líneas se pueden desvelar a partir de la revisión realizada.

Las condiciones y modalidades de aprendizaje están cambiando constantemente y será necesario abordar estudios en profundidad de los procesos de reenganche que den cuenta de las condiciones en que se desarrollan y su mayor o menor grado de éxito, a la vez que consideran las experiencias de los participantes en los mismos. Será preciso abordar, tanto los procesos de desenganche como de los de reenganche, desde una perspectiva más holística y a medio plazo, que considere las trayectorias globales de los sujetos y no momentos aislados de su recorrido. Inevitablemente, dado que el concepto de desenganche está relacionado, a su vez, al de "éxito educativo", es ineludible someter a este último a una revisión crítica que tenga en cuenta las necesidades de desarrollo individual y social de los escolares, así como el propio sentido de la educación obligatoria. Por ello, deberán manejarse visiones más comprehensivas de la eficacia educativa que abarquen el conjunto de dimensiones del desarrollo humano, más allá de las estrictamente académicas o laborales.

Sea como fuere, las mejoras en este terreno han de plantearse con una mirada multidimensional, sistémica y profunda, más allá de meras acciones simbólicas y superficiales que no alteran modos de ser, hacer o funcionar que han de ser removidos y transformados. Los centros escolares, sugieren Down y otros (2014, p. 9), han de ser "reculturizados, re-estructurados y re-diseñados de forma que conecten con las vidas, lenguajes, experiencias, necesidades de los estudiantes". No caben soluciones simplistas, descontextualizadas y unidimensionales a problemas tan complejos.

\section{Referencias}

ALA. (2014). Disengaged youth and ACE. Adult Learning Australia. Recuperado de https://ala.asn.au/ 
Aristimuño, A. y Parodi, P. (2017). Un caso real de combate al fracaso en la educación pública: Una cuestión de acompañamiento, liderazgo y cultura organizacional. REICE. Revista Iberoamericana sobre Calidad, Eficacia y Cambio en Educación, 15(4), 141-157. https://doi.org/10.15366/reice2017.15.4.008

Ashwin, P. y McVitty, D. (2016). The meanings of student engagement: Implications for policies and practices. En A. Curaj (Ed.). The European higher education area (pp. 343-359). Nueva York, NY: Springer. https://doi.org/10.1007/978-3-319-20877-0_23 343-359

Atweh, B., Bland, D., Carrington, S. y Cavanagh, R. (mayo, 2007). School disengagement: Its constructions, investigation and management. Comunicación presentada en el AARE 2007 International Educational Research Conference. Geelong, Deakin University.

Blaya, C. y Bergamaschi, A. (2015). L'abbandono scolastico in Francia. Analisi critica delle politiche pubbliche. Scuola Democrattica, 2, 425-437.

Bond, L., Butler, H., Thomas, L., Carlin, J., Glover, S., Bowes, G. y Patton, G. (2007). Social and school connectedness in early secondary school as predictors of late teenage substance use, mental health, and academic outcomes. Journal of Adolescent Health, 40, 9-18. https://doi.org/10.1016/j.jadohealth.2006.10.013

Callanan, M., Kinsella, R., Graham, J., Turczuk, O. y Finch, S. (2009). Pupils with declining attainment at key stages 3 and 4: Profiles, experiences and impacts of underachievement and disengagement. Londres: DCSF.

CEDEFOP. (2010). Guiding at-risk youth learning to work: Lessons from across Europe. Luxemburgo: Office of the European Union.

Coffield, F. y Edward. S. (2009). Rolling out "good", "best” and "excellent practice. What next? Perfect practice? British Educational Research Journal, 35(3), 371-390. https://doi.org/10.1080/0141 1920802044396

Comisión Europea. (2013). Reducing early school leaving: Key messages and policy support. Luxembugo: Publications Office of the European Union.

Comisión Europea. (2014). Tackling early leaving from education and training in Europe: Strategies, policies and measures. Luxembugo: Publications Office of the European Union.

Conner, T. (2016). Relationships: The key to student engagement. International Journal of Education and Learning, 5(1), 13-22. https://doi.org/10.14257/ijel.2016.5.1.02

Davies, M., Lamb, S. y Doecke, E. (2011). Strategic review of effective re-engagement models for disengaged learners. Victoria: Victorian Department of Education and Early Childhood Development.

Day, L. (2013). Preventing early school leaving in Europe. Lessons learned from second chance education. Luxembugo: European Commission.

Down, B., Smyth, J., Robinson, J. y McInerney, P. (2014). Rethinking the conditions for young people getting a job. Kids have something to say. Perth: Murdoch University.

Downes, P., Nairz-Wirth, E. y Rusinaitè, V. (2016). Structural indicators for inclusive systems in and around schools. Luxembugo: Publications Office of the European Union.

Echeita, G. y Sandoval, M. (2010). Claves de la equidad como reto de la educación del siglo XXI. En G. Echeita, M. Sandoval, N. Illán, J. Domingo, M. Soler, N. Melero y F. Luengo (Coords.), Inclusión y educación democrática: Éxito para todos y todas a lo largo de la vida (pp. 717). Madrid: Proyecto Atlántida.

Escudero, J. M. (2013). Estudiantes en riesgo, centros escolares de riesgo: Respuestas educativas al alumnado en situaciones de vulnerabilidad. Murcia: Diego Marín. 
Escudero, J. M. (2016). Inclusión y exclusión educativa: Realidades, miradas y propuestas. Valencia: Nau Llibres.

Escudero, J. M. y Martínez, B. (2012). Las políticas de lucha contra el fracaso escolar: ¿Programas especiales o cambios profundos del sistema y la educación? Revista de Educación, 359, 174193.

Evans, J., Meyer, D., Pinney, A. y Robinson, B. (2009). Second chances: Re-engaging young people in education and training. Essex: Barnardo`s.

Fernández Enguita, M., Mena, L. y Riviére, J. (2010). Fracaso y abandono escolar en España. Barcelona: La Caixa.

Fullan, M. y Longworthy, M. (2014). A rich seam: How new pedagogies find deep learning. Harlow: Pearson.

González, M. T. (2010). El alumno ante la escuela y su propio aprendizaje: Algunas líneas de investigación en torno al concepto de implicación. REICE. Revista Iberoamericana sobre Calidad, Eficacia y Cambio en Educación, 8(4), 10-31.

González, M. T. (2015a). Los centros escolares y su contribución a paliar el desenganche y abandono escolar. Profesorado. Revista de Currículum y Formación del Profesorado, 19(3),158176.

González, M. T. (2015b). La vulnerabilidad escolar y los programas de cualificación profesional inicial. Madrid: Wolters Kluwer.

González, M. T. (2016). Una mirada al pasado más reciente: El fracaso escolar y los intentos de acometerlo a base de programas extraordinarios de atención a la diversidad. En J. M. Escudero (Comp.), Inclusión y exclusión educativa: Realidades, miradas y propuestas (pp. 115134). Valencia: Nau Llibres.

González, M. T. y Currás, M. (2013). ¿Hacer más de lo mismo en las aulas conduce a un mayor engagement? El caso del programa de cualificación profesional inicial en la Región de Murcia. En F. Veiga (Coord.), Envolvimento dos alunos na escola: Perspetivas internacionais da psicologia e educação (pp. 795-815). Lisboa: Universidade de Lisboa.

Gasch, B. y Navas, A. (2017). Expulsados del sistema. Cuadernos de Pedagogía, 478, 1-6.

Hancock, K. J. y Zubrick, S. R. (2015). Children and young people at risk of disengagement from school. Crawley: Commissioner for Children and Young People WA.

Ingólfur, J. y Valgerður, S. B. (2016). Meaningful education for returning-to-school students in a comprehensive upper secondary school in Iceland. Critical Studies in Education, 57(1), 56-87.

Johns, N. (2014). Flexible and alternative education: Hearing the voices of young people. Melbourne: Universidad de Melbourne.

Kettlewell, K., Southcott, C., Stevens, E. y McCrone, T. (2012). Engaging the disengaged. Slough: NFER.

Knesting, K. (2008). Students at risk for school dropout: Supporting their persistence. Preventing School Failure, 52(4), 3-10. https://doi.org/10.3200/PSFL.52.4.3-10

Lago, M., Gómez, C., Vidal, R. A., Fernández, A. y Soto, J. (2015). Una alternativa al fracaso escolar: Escuelas de segunda oportunidad. Londres: Lulu press.

Li, Y. (2017). Teacher-student relationships, student engagement, and academic achievement for non-latino and latino youth. Adolescent Research Review, 9(3), 1-50. https://doi.org/10.1007/s40894-017-0069-9 
Lloyd-Jones, S., Bowen, R., Holton, D., Griffin, T. y Sims, J. (2010). A qualitative research study to explore young people's disengagement from learning. Gales: Welsh Assembly Government Social Research.

Lumby, J. (2013). Education isn't working for us listening to disengaged young people. Bera Insights, 5, 1-4.

Magalhaes, A. M., Araujo, H. C., Macedo, E. y Rocha, C. (2015). Early school leaving in Portugal: Policies and actors' interpretations. Educaçao Sociedade \& Culturas, 45, 97-120.

Maroulis, S. y Gomez, L. M. (2008). Does “connectedness” matter? Evidence from a social network analysis within a small-school reform. Teachers College Record, 110(9), 1901-1929.

McMahon, B., Munns, G., Smyth, J. y Zyngier, D. (2012). Student engagement for equity and social justice: creating space for student voice. Teaching $\mathcal{E}^{2}$ Learning, 7(2), 63-78. https://doi.org/10.26522/tl.v7i2.417

McLaughlin, C. y Gray, J. (2015). Adolescent well-being and the relational school. En C. Mclaughlin (Ed.), The connected school. A design for well-being. Supporting children and young people in schools to flourish, thrive and achieve (pp. 1-7). Londres: Pearson.

MECD. (2014). Plan para la reducción del abandono educativo temprano. Recuperado de http://www.mecd.gob.es

Mena, L., Fernández Enguita, M. y Riviére, J. (2010). Desenganchados de la educación: Procesos, experiencias, motivaciones y estrategias del abandono y del fracaso escolar. Revista de Educación, 352, 119-145.

Menzies, L. y Baars, S. (2015). The alternative should not be inferior. What now for 'pushed out' learners? Recuperado de https://cdn.lkmco.org/

Mills, M. y McGregor, G. (2010). Re-engaging students in education success factors in alternative schools. routh affairs network of Queensland. Queensland: Queesland Government.

Morentin, J. y Ballesteros, B. (2018). Desde fuera de la escuela: Una reflexión en torno al aprendizaje a partir de trayectorias de abandono escolar prematuro. REICE. Revista Iberoamericana sobre Calidad, Eficacia y Cambio en Educación, 16(1), 5-20. https://doi.org/10.15366/reice2018.16.1.001

Murray, S. y Mitchell, J. (2016). Teaching practices that re-engage early school leavers in further education: An Australian study. Journal of Further and Higher Education, 40(3), 372-391. https://doi.org/10.1080/0309877X.2014.971107

Murray-Harvey, R. y Slee, P. T. (2007). Supportive and stressful relationships with teachers, peers and family and their influence on students' social/emotional and academic experience of school. Australian Journal of Guidance \& Counselling, 17(2), 126-147. https://doi.org/10.1375/ajgc.17.2.126

Nelson, J. (2011). Strategies to re-engage young people not in education, employment or training. A rapid review. Londres: Centre for Excellence and Outcomes in Children and Young People's Services.

Nelson, J. y O'Donnell, L. (2012). Approaches to supporting young people not in education, employment or training: A review. Londres: Centre for Excellence and Outcomes in Children and Young People's Services.

Nouwen, W., Van Praag, L., Van Caudenberg, R., Clycq, N. y Timmerman, C. (2016). School-based prevention and intervention measures and alternative learning approaches to reduce early school leaving. Amberes: European Commission. 
Paul Hamlyn Foundation. (2012). The engaging school: A handbook for school leaders. Londres: The Innovation Unit

Portelli, J. P., Shields, C. M. y Vibert, C. B. (2007). Toward an equitable education: Poverty, diversity and students at-risk. Toronto: OISE.

Rambla, X. y Fontdevila, C. (2015). La política de la Unión Europea y del gobierno de España contra el abandono escolar prematuro. En A. Tarabini (Coord.), Políticas de lucha contra el abandono escolar en España (pp. 13-24). Madrid: Síntesis.

Riele, K. (2014). Putting the jigsaw together: Flexible learning programs in Australia. Melbourne: The Victoria Institute for Education, Diversity and Lifelong Learning.

Ross, A. (2009). Disengagement from education among 14-16-year old. Londres: National Centre for Social Research.

Rumberger, R. W. (2011). Dropping out: Why students drop out of high school and what can be done about it. Cambridge, MA: Harvard University Press. https://doi.org/10.4159/harvard.9780674063167

Rumberger, R., Addis, H., Allensworth, E., Balfanz, R., Bruch, J., Dillon, E., ... y Tuttle, C. (2017). Preventing dropout in secondary schools. Washington, DC: U.S. Department of Education.

Salvà, F., Trobat, M. F. y Forgas, R. (2014). Abandono escolar y desvinculación de la escuela: Perspectiva del alumnado. Magis, Revista Internacional de Investigación en Educación, 6(13), 129-142. https://doi.org/10.11144/Javeriana.M6-13.aede

Seguedin, L. (2012). Listening to the student voice: Understanding the school-related factors that limit student success. McGill Journal of Education, 47(1), 93-107. https://doi.org/10.7202/1011668ar

Shoda, S. y Guglielmi, S. (2009). A stitch in time: Tackling educational disengagement interim report. Londres: Gotham Rounded.

Smyth, J. (2006). When students have power: Student engagement, student voice, and the possibilities for school reform around 'dropping out' of school. International Journal of Leadership in Education, 9(4), 285-298. https://doi.org/10.1080/13603120600894232

Smyth, J. (2012). When students "speak back”. Student engagement towards a socially just society. En B. J. McMahon y J. P. Portelli, (Eds.), Student engagement in urban schools. Beyond neloberal discourses (pp. 73-90). Charlote, NC: Information Age Publishing.

Smyth, J., Angus, L., Down, B. y Mcinerney, P. (2008). Critically engaged learning. Connecting to young lives. Nueva York, NY: Peter Lang Publishng.

Spielhofer, T., Benton, T., Evans, K., Featherstone G., Golden, S., Nelson, J. y Smith, P. (2009). Increasing participation. Understanding young people who do not participate in education or training at 16 and 17. Londres: National Foundation for Educational Research.

Stamou, E., Edwards, A., Daniels, H. y Ferguson, L. (2014). Toung people at-risk of drop-out from education: Recognizing and responding to their needs. Oxford: University of Oxford.

Strobel, K., Kirshner, B., O’Donoghue, J. y McLaughlin, M. (2008). Qualities that attract urban youth to after-school settings and promote continued participation. Teachers College Record, $110(8), 1677-1705$.

Suárez, C., Pimentel, A. y Martin, M. (2009). The significance of relations. Academia engagement and achievement among newcomer inmigrant youth. Teacher College Record, 111 (3), $712-$ 749 . 
Tadich, B., Deed, C., Campbell, C. y Prain, V. (2007). Student engagement in the middle years: A year 8 case study. Issues in Educational Research, 17(2), 256-271.

Tarabini, A. (2015). Políticas de lucha contra el abandono escolar en España. Madrid: Síntesis.

Tomaszewska-Pekala, H., Marchlik, P. y Wrona, A. (2015). Between school and work. Vocational education and the policy against early school leaving in Poland. Educaçao Sociedade E゚ Culturas, 45, 75-96.

Traag, T. y Van der Velden, R. K. W. (2008). Early school-leaving in the Netherlands. The role of student, family and school factors for early school-leaving in lower secondary education. Maastricht: Research Centre for Education and the Labour Market.

Trowler, V. y Trowler, P. (2010). Student engagement evidence summary. Lancaster: University of Lancaster.

Thureau, G. y Usón, J. M. (2017). Las E2O en el mundo. Cuadernos de Pedagogía, 478, 1-24.

Wilson, K., Stemp, K. y McGinty, S (2011). Re-engaging young people with education and training. What are the alternatives? Touth Studies Australia, 30(4), 32-49.

Wogboroma, O. (2014). Investigating factors that support re-engagement of 14-16 year olds in education. Open Access Library Journal, 1, 904-92 1. https://doi.org/10.4236/oalib.1 100904

Zyngier, D. (2007). (Re)conceiving student engagement: What the students say they want. Putting young people at the centre of the conversation. Learning Landscapes, 1(1), 93-116.

Zyngier, D. (2012). Transforming student engagement. An empowering pedagogy: An Australian experience in the classroom. En B. J. McMahon (Ed.), Student engagement in urban schools: Beyond neoliberal discourses (pp. 111-132). Toronto: Information Age Pub.

Zyngier, D. y Gale, T. (marzo, 2003). Engaging programs: How are Australian schools responding to low student retention? Comnicación presentada en el AARE International Conference. Auckland New Zealand.

\section{Breve CV de los autores}

\section{$\mathbf{M}^{\mathrm{a}}$ Teresa González González}

Profesora Titular de Organización Escolar en la Facultad de Educación de la Universidad de Murcia. Miembro del grupo de investigación Equidad e Inclusión Educativa de dicha universidad, y de la Red de Investigación sobre Liderazgo y Mejora de la Educación. Su trayectoria de investigación y publicaciones abarca varias líneas de trabajo: a) Las escuelas como organización: Estructuras para la coordinación docente; Agrupamientos; Relaciones profesionales y micropolíticas; Cultura organizativa; Procesos de dirección; liderazgo pedagógico; Mejora escolar, etc. b) Alumnado en riesgo de exclusión educativa: Factores de riesgo; absentismo escolar y posibles respuestas desde los centros escolares, desenganche o desapego escolar, medidas y programas para paliar el desapego y evitar el abandono temprano, etc. ORCID ID: 0000-0002-3059-7173. Email: mtgg@um.es

\section{José Luis San Fabián Maroto}

Catedrático de Universidad de Didáctica y Organización Escolar. Titulado en Pedagogía y Sociología. Investiga en los campos de Organización y Evaluación de Programas e Instituciones Educativas, Estudios sobre la Infancia, Investigación cualitativa en Educación. Ha publicado numerosos trabajos en revistas científicas. Ha dirigido 21 tesis 
doctorales. Obtuvo el Premio extraordinario de Doctorado por la Universidad Complutense de Madrid. Ha participado en más de 30 proyectos de investigación. Es director del Programa de Doctorado en "Equidad e innovación educativa" de la Universidad de Oviedo. Ha dirigido el Departamento de Ciencias de la Educación. Ha sido director de la Revista Organización y Gestión Educativa, del Forum Europeo de Administradores de la Educación. Dirige el grupo de investigación "Análisis, Intervención y Evaluación Socioeducativa" (AIES) (http://aies.grupos.uniovi.es/). ORCID ID: 0000o002-8149-7797.Email: jlsanfa@uniovi.es 